\title{
Full-field X-ray Nano-scope Developed at SSRF
}

Biao Deng ${ }^{1}$, Yudan ${ }^{1}$ Wang, Yuqi Ren ${ }^{1}$,Tianxi Sun ${ }^{2}$, Guangzhao ${ }^{1}$ Zhou,Guohao Du ${ }^{1}$, Fen Tao ${ }^{1}$, Honglan $\mathrm{Xie}^{1}$, Tiqiao Xiao ${ }^{1}$

1. Shanghai Institute of Applied Physics, CAS, Shanghai, China

2. Beijing Normal University, Beijing, China

Full-field X-ray nano-scope is one of the most powerful tools for in-situ, non-destructive observation of the inner structure of objects with high resolution. Many X-ray nano-scope systems have been constructed in the world and have realized 30nm spatial resolution with 20 microns field of view(FOV). Based on the user operation experiences of years at Shanghai Synchrotron Radiation Facility(SSRF) X-ray imaging beamline, we get to know that lots of user experiments will rely on X-ray full-field nano-CT with big FOV and $100 \mathrm{~nm}$ scale resolution. A full-field X-ray nano-imaging system has been designed and constructed at SSRF [1]. The microscope is based on a beam shaper and a zone plate using both absorption contrast and Zernike phase contrast, with the optimized energy set to $10 \mathrm{keV}$. The experimental test results by a Siemens star pattern demonstrate that a spatial resolution of $100 \mathrm{~nm}$ is achieved, while an FOV of $50 \mu \mathrm{m}$ is obtained [2]. And a full-field X-ray nano-CT system based Equally Sloped Tomography(EST) was developed. 3D imaging of tantalum particles reconstructed by EST with 128 projections was reported [3]. The nano-scope is ready to be opened to users. And a dedicated full field X-ray nano-imaging beamline based on bending magnet is under construction in the SSRF phase-II project. The beamline aims at the 3D imaging of the nano-scale inner structures. The photon energy range is of 5-14keV. The design goals with the FOV of 20 microns and a spatial resolution of $20 \mathrm{~nm}$ are proposed at $8 \mathrm{keV}$.

In full-field X-ray nano-imaging, the most commonly used condenser devices are zone plates and ellipsoidal monocapillaries. In contrast, the transmission efficiency of ellipsoidal glass monocapillary is higher than that of zone plates. Therefore, ellipsoidal monocapillaries are generally adopted as focusing lenses in full-field transmission X-ray nano-imaging. An ellipsoidal glass monocapillary was designed and fabricated and its performance was measured by both optical measurement and synchrotron radiation X-ray test. The monocapillary had slope errors as low as $15 \mu \mathrm{rad} \mathrm{rms}$. The images of the focal spot and the far-field pattern recorded by X-ray detector showed that the focusing size was $40 \mu \mathrm{m}$ and divergences was $1.86 \mathrm{mrad}$ at $9 \mathrm{kev}$. Based the monocapillary, a full Field Transmission X-ray Microscopes(TXM) System was designed and constructed at SSRF. The spatial resolution of $100 \mathrm{~nm}$ was achieved by utilizing the monocapillary condenser [4,5], which indicating the ellipsoidal monocapillary had high quality and could meet the requirement of X-ray nano-imaging. In the future, a new monocapillary will be fabricated for and used as condenser in SSRF TXM bemline for 20nm TXM.

References:

[1] Biao Deng et al. SPIE, 8851 (2013), p. 88511D-1

[2] Binggang Feng et al. CHINESE OPTICS LETTERS 14 (2016), p. 093401.

[3] Yudan Wang, Yuqi Ren, Guangzhao Zhou, et al, (submitted)

[4] Fen Tao, Yudan Wang, Yuqi Ren, et al. ACTAOPTICASINICA 37 (2017), p. 0034002.

[5] Bowen Jiang, Zhiluo Liu, Xuepeng Sun, et al. Optics Communications 398 (2017), p. 91. 

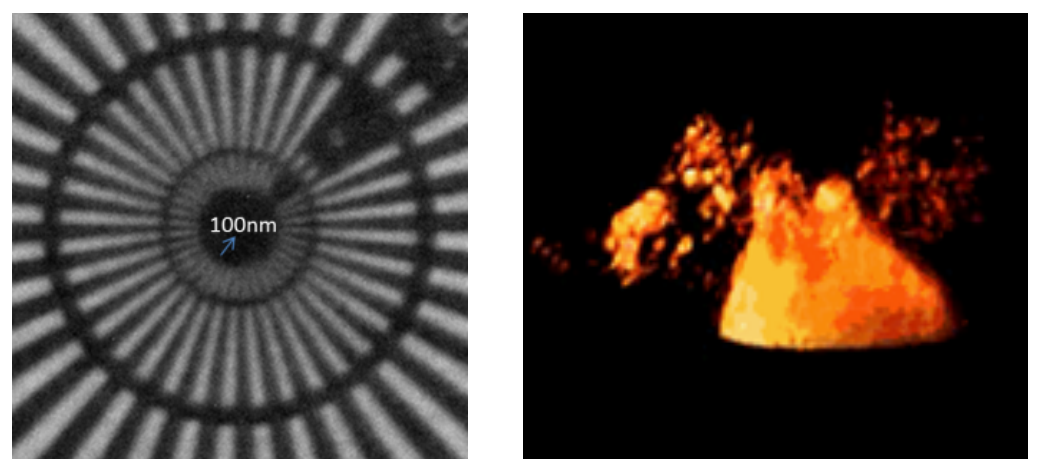

Figure 1. The TXM imaging of resolution target Figure 2. 3D imaging of particles based EST
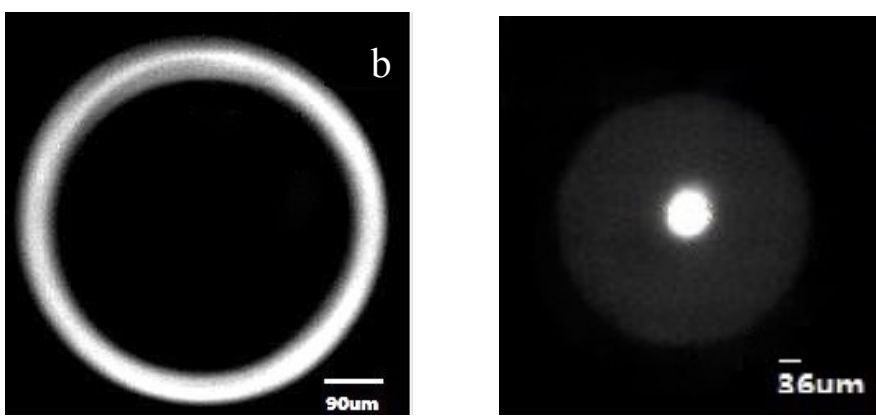

Figure 3. (a) The far-field pattern of the monocapillary; (b) The focused spot

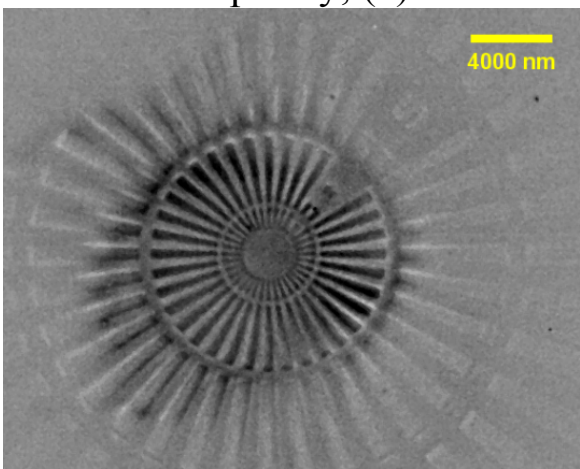

Figure 4. The TXM imaging of $100 \mathrm{~nm}$ resolution target 